\title{
The relation between rage polymorphism and cardiovascular diseases: a review
}

\begin{abstract}
RAGE [receptor for AGEs (advanced glycation end-products)] plays an important role in the development and progression of vascular disease. Studies in cultured cells and small animal models of disease have clearly demonstrated that RAGE is central to the pathogenesis of vascular disease of the macro- and micro-vessels in both the diabetic and non-diabetic state. Emerging results from human clinical studies have revealed that levels of circulating soluble RAGE in the plasma may reflect the presence and/ or extent of vascular disease state. Additionally, genetic variants of the RAGE gene (AGER in HUGO nomenclature) have been associated with vascular disease risk. Combining RAGE circulating protein levels and the presence of particular RAGE polymorphisms may be a useful clinical tool for the prediction of individuals at risk for vascular disease. Therapeutic intervention targeted at the RAGE gene may therefore be a useful means of treating pathologies of the vasculature. The receptor for advanced glycation end-products (RAGE) is expressed to enhance degrees in human atherosclerotic plaques and colocalizes with inflammatory and pro-oxidant mediators in the vulnerable regions of the plaque. Previous studies highlighted a number of variants in the gene encoding the receptor, including a Gly to Ser substitution at amino acid 82 within the ligand-binding domain of RAGE. The Ser 82 allele enhanced ligandbinding affinity and increased ligand-stimulated generation of inflammatory mediators in transfected cells and human monocytes compared to the common RAGE Gly82 allele. Further studies are required on other more prevalent genetic variants of RAGE and cardiovascular disease.
\end{abstract}

Keywords: heart failure, diabetes, receptor for advanced glycation end-products (rage), polymorphisms, stroke, susceptibility, inflammation
Volume 2 Issue 2 - 2017

\author{
Deeraj Mungun,' Iyan Zakaria,' Xiang Ming \\ Wan,' Lu Miao,' Tian Tian Tu,' Yan Guo',2 \\ 'Department of Geriatric Cardiology, First Affiliated Hospital of \\ Nanjing Medical University, China \\ ${ }^{2}$ Department of Cardiology, Shengze Hospital of Jiangsu \\ Province, China
}

Correspondence: Yan Guo, Department of Geriatric Cardiology, Department of Cardiology, First Affiliated Hospital of Nanjing Medical University, Nanjing, China, Email guoyan5I@hotmail.com

Received: August 12, 2017 | Published: September 13, 2017
Abbreviations: AGE, advanced glycation end-product; ANGII, angiotensin ii; CAD, coronary artery disease; Dia-1, diaphanous-1; DN, dominant-negative; Hbalc, glycated haemoglobin; HMGB1, high-mobility group box-1; LTA, lymphotoxin A; NF-Kb, nuclear factor kb; NMD, nonsense-mediated mrna-decay; PBX2, Pre-B-cell leukaemia homeobox 5; RAGE, receptor for ages; Ragev1, rage splice variant 1; SNP, single nucleotide polymorphism; Srage, soluble rage; Esrage, endogenous srage; TNF, tumour necrosis factor; UTR, untranslated region

\section{Introduction}

The receptor for advanced glycation end-products (RAGE) is a multiligand member of the immunoglobulin superfamily of cell surface molecules. Although first described as a receptor for AGEs (advanced glycation end- products), more recent studies suggest that RAGE interacts with proinflammatory ligands, including members of the S100/calgranulin family and amphoterin. ${ }^{1}$ Engagement of RAGE by these ligands upregulate expression of inflammatory and prothrombotic molecules thereby suggesting a link between the receptor and cardiovascular disease. Recent studies demonstrated that significantly higher levels of RAGE are seen in human atherosclerotic plaque. $^{2,3}$

RAGE expression was found to co-localize particularly in macrophages at the vulnerable regions of the atherosclerotic plaque with inflammatory mediators. To date, the pathogenic role of RAGE in accelerated diabetic atherosclerosis has been suggested by the results of studies in murine models. In mice deficient in apolipoprotein E (apo E) rendered diabetic with streptozotocin, accelerated atherosclerotic lesion area and complexity was reduced by administration of soluble (s) RAGE, the extracellular ligand-binding omain of RAGE. ${ }^{4}$ Furthermore, administration of sRAGE to nondiabetic apo E null mice also significantly attenuated atherosclerosis, albeit to lesser degrees than that observed in the diabetic mice. These data highlight RAGE as a candidate gene for study in vascular disease, particularly in the setting of diabetes. Our previous studies have highlighted a number of variants including a Gly to Ser substitution at amino acid number 82, which lies within the ligand-binding domain of RAGE. ${ }^{5}$ The Ser82 isoform has been shown in in vitro studies to display higher ligand affinity in transfected cells versus the common Gly82 isoform of RAGE. Further, in both transfected CHO cells and human monocytes naturally expressing the Ser82 isoform of RAGE, increased ligand-stimulated activation of mediators linked to inflammation and vascular disease was observed compared to cells expressing the common Gly82 isoform. ${ }^{6}$ Small studies of UK and Finnish Caucasians subjects with cardiovascular disease revealed no association with the Gly82Ser polymorphism, however, a number of studies demonstrate a possible association of this allelic variant with other vascular complications of diabetes Interestingly, the Gly82Ser polymorphism is in linkage disequilibrium with HLA DR4 and when assessed in rheumatoid arthritis an HLA DR4-associated disorder, an increased prevalence of the Ser 82 allele was seen in subjects with RA compared to control subjects. ${ }^{7}$

Heart failure (HF) is a clinical syndrome that represents the endstage of several heart diseases and remains a major cause of morbidity and mortality worldwide. Despite recent advances in its medical treatment, mortality rates due to HF remain elevated. ${ }^{8}$ 
The receptor for advanced glycation end-products (RAGE) is a member of the immunoglobulin superfamily of cell surface proteins that binds advanced glycation end-products (AGEs) and other molecules. It is expressed at low levels in most adult tissues under physiological conditions, but is overexpressed in the vessel wall at sites of vascular pathology. ${ }^{9}$ In particular, RAGE levels are higher in diabetic patients and animal models of diabetes than in their nondiabetic counterparts ${ }^{9,10}$ Accumulating experimental evidence has shown that the interaction of AGEs with RAGE leads to activation of intracellular signaling, gene expression, and production of proinflammatory cytokines and free radicals, thus playing a central role in the pathogenesis of vascular and heart disease ${ }^{5,10}$ sRAGE, the soluble form of RAGE, can be formed by both alternative splicing of the RAGE gene (endogenously secreted RAGE-esRAGE) and proteolytic cleavage of membrane-bound RAGE (cleaved RAGE$c R A G E$ ). The ability of sRAGE to bind RAGE ligands suggests a cytoprotective action against AGEs. ${ }^{11}$ Two prospective studies showed that high sRAGE circulating levels were associated with severity of disease and predicted cardiac events in patients with HF, regardless of AGE levels and other known prognostic parameters.

Two prospective studies showed that high sRAGE circulating levels were associated with severity of disease and predicted cardiac events in patients with HF, regardless of AGE levels and other known prognostic parameters. ${ }^{12,13}$ Moreover, high sRAGE levels were also associated with ischemic $\mathrm{HF}^{14}$ and one study has shown that cRAGE levels are increased while esRAGE levels are decreased in patients with HF as compared to healthy controls ${ }^{15}$ From this study, we can learn about RAGE polymorphism and comparasion between different cardiovascular polymorphism.

\section{Receptor for advanced glycation end- products(RAGE)}

Advanced Glycation End-products (AGEs) are a heterogeneous and complex group of biochemical modifications, which play an important role in development of chronic disease processes. AGEs cause a wide range of deleterious effects, which are mediated by cellular receptor, especially RAGE (Receptor for Advanced Glycation End-products). RAGE, a multi-ligand member of the immunoglobulin superfamily is a ubiquitous receptor present on epithelial, neuronal, vascular and inflammatory cells, usually expressed at low levels in homeostasis and to increased degrees at sites of stress or injury. RAGE acts as a pattern recognition receptor for ligands released by inflamed, stressed and damaged cells. Increased expression of both cell-surface RAGE and accumulation of its ligands was observed in a range of disorders characterized by chronic inflammation, such as inflammatory bowel disease, rheumatoid arthritis, atherosclerosis, amyloidoses, Alzheimer's disease and the vascular complications of diabetes. RAGE is composed of a large extracellular part, a transmembrane domain and a 43-amino-acid-long cytoplasmic tail. The extracellular domain includes a short signaling sequence for the cell membrane binding and two C-type and one V-type immunoglobinlike domains. The ligand-binding site is into V-domain, whereas the cytosolic tail mediates intracellular signaling. More recently, it has been suggested that RAGE may form dimers and multimers for ligand binding to occur. ${ }^{7}$

A range of splice variants of murine and human RAGE has been identified; most of these lead to removal of the transmembrane domain to produce soluble variants. RAGE has a C-truncated secretory isoform that circulates in plasma and which at least two variants: one secreted from cells, endogenously secreted RAGE (esRAGE), and another one, which formed by proteolytic cleavage, by matrix metalloproteinases from the cell-surface, cleaved-RAGE (cRAGE). The soluble isoform (sRAGE) acts as a decoy receptor for RAGE ligands, and is thought to afford protection against inflammation. The RAGE-ligand interaction triggers activation of NF- $\mathrm{kB}$ and other signaling pathways through stimulation of p21ras, ERK (extracellular signal-regulated kinase) 1/2, p38 MAPK, SAPK (stress-activated protein kinase)/JNK (c-Jun n-terminal kinase), Rho GTPases, PI3K and JAK (Janus kinase)/ STAT (signal transducer and activator of transcription) pathways ${ }^{16}$ ubsequently, expression of inflammatory cytokines increased, leading to an inflammatory response with associated cellular migration and proliferation. Ligation of RAGE causes a positive feed-forward loop in which inflammatory stimuli activate NF- $\mathrm{KB}$, which induces RAGE expression, followed again by NF- $\kappa \mathrm{B}$ activation. NF- $\kappa \mathrm{B}$ up-regulates multiple cellular signaling cascades and determines increased production of numerous growth factors and cytokines. ${ }^{17}$

The complexity of the role of RAGE in the biological setting is also evident from human studies in different vascular disease states. In patients with heart failure, higher sRAGE concentration and RAGE -374T/A polymorphism in RAGE gene promoter was associated with heart failure severity. On the other hand, many studies have pointed out that decreased sRAGE levels are associated with increased extent of CAD and with higher incidence of cardiovascular events, such as myocardial infarction. Falcone $\mathrm{C}$ et al. in 2005 , showed that low plasma levels of sRAGE were independently associated with the prevalence of CAD in non-diabetic men. Recent data from the Dallas Heart Study ${ }^{18}$ confirm that lower levels of sRAGE are independently associated with a greater prevalence of coronary atherosclerosis. More recently, Falcone et al., demonstrated that sRAGE plasma levels were significantly lower in patients with ACS than in patients with stable angina, suggesting that SRAGE can be considered an indicator of destabilization of vulnerable plaque. ${ }^{19}$ In addition, it shown that CAD patients presenting with peripheral artery disease have lower sRAGE levels than CAD patients without peripheral atherosclerosis, showing that stable atherosclerotic lesions in different vascular districts are inversely related to soluble decoy receptor sRAGE. ${ }^{20}$ Moreover, it was shown that certain polymorphisms in the RAGE gene (-374T/A, $-429 \mathrm{~T} / \mathrm{C}, \mathrm{G} 82 \mathrm{~S}$ ) are strongly associated with higher sRAGE levels, implicating a complex genetic regulation of sRAGE levels and suggesting that sRAGE may not be a marker of a disease state but also a potential target in the pathobiology of the atherosclerotic process. The correlation between the RAGE gene polymorphisms and CAD was extensively investigated and several studies demonstrated that $-374 \mathrm{~A}$ allele might reduce susceptibility to CAD exerting a protective effect on coronary risk. ${ }^{21}$ In a study of Falcone $\mathrm{C}$ et al, the mean number of injured vessels was significantly lower in AA genotype than AT or TT genotype patients and it would seems to indicate that this polymorphism is one of the likely determinants candidate for genetic variance of disease phenotype in coronary atherosclerosis . Another study by the same research group indicated that $-374 \mathrm{~T} / \mathrm{a}$ polymorphism could be also associated with a reduced risk of in-stent restenosis after coronary stent implantation. These findings clearly illustrate the need for further studies of the complex involvement of RAGE in the development of vascular disease states., ${ }^{4,22}$

\section{Structure and variants of rage}

RAGE is an approximately $45 \mathrm{kDa}$ protein, originally isolated 
from bovine lung endothelium on the basis of its ability to bind advanced glycation end products and later has been characterized as a member of the immunoglobulin (Ig) superfamily of cell-surface molecules. The entire mature receptor consists of 403 amino acids in man, rat, and mouse ${ }^{23}$ he extracellular region of RAGE consists of one V-type(variable) immunoglobulin domain, followed by two C-type (constant)immunoglobulin domains stabilized by internal disulfide bridges between cysteine residues. ${ }^{24}$

The existence of diverse RAGE isoforms from the same gene indicates that the pre-mRNA of RAGE undergoes alternative splicing. In vitro studies have shown that N-truncated isoform of RAGE is expressed on the cell surface in a way that is similar to full-length RAGE. The V domain of RAGE has been shown to be critical for ligand binding; the N-truncated RAGE is unable to engage glycated end products. Nonetheless, it has been suggested that N-truncated RAGE could participate in the regulation of angiogenesis in a way that is independent from the classical RAGE activation pathway ${ }^{25}$ (Figure 1)

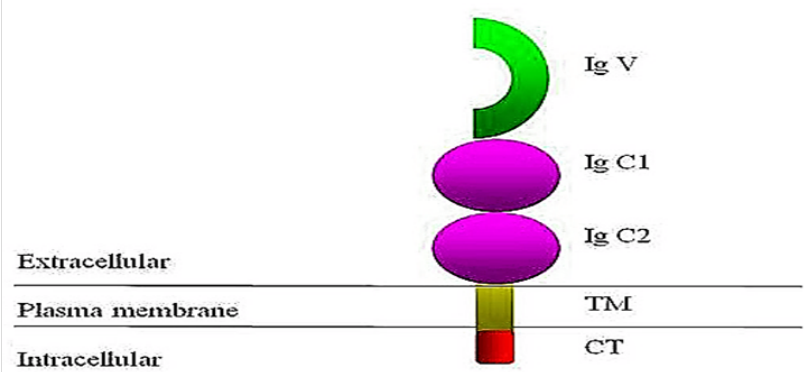

Figure I RAGE protein structure and organization. Schematic drawing of the RAGE protein showing domains and extracellular, transmembrane and intracellular organization

Human RAGE gene was mapped in chromosome 6p21.3, located in the main histocompatibility complex between class II and III regions (GenBank/EMBL - Data Bank Accession Number D28769). This gene comprises $1.7 \mathrm{~kb}, 11$ exons and 10 introns of variable size in 3'UTR region, which may reach $4 \mathrm{kpb}$. Moreover, immunoglobulin type $\mathrm{V}$ domain is codified by the second and third exons. ${ }^{1}$

Over 50 genetic variants have been described for RAGE gene. The functional single nucleotide polymorphisms (SNPs), including variation I codon 82 (G82S, rs 2070600), resulting from the amino acid exchange of glycine for serine in domain $\mathrm{V}$ and two polymorphic regions in the promoting region $-429 \mathrm{~T}>\mathrm{C}$ ( $\mathrm{rs}$ 1800625) and $-374 \mathrm{~T}>\mathrm{A}$ (rs 1800624), have been extensively researched in association with pathologies. ${ }^{26}$ The allele $374 \mathrm{~A}$ of RAGE promoter has been associated as a protector against macro vascular damage in patients with DM type 2 and the allele $-429 \mathrm{C}$ with diabetes type 1 (DM1). More recently, the genome-wide association studies (GWAS) has shown the association of polymorphism G82S with pulmonary function. Other polymorphism associations with RAGE gene have also been reported: breast tumor, pancreas tumor, gastric tumor, multiple sclerosis and diabetic complications. ${ }^{11}$

\section{Mechanisms of cardiovascular disease: atherosclerosis}

Atherosclerosis is by far the most important underlying pathologic process for cardiovascular disease. Therefore, an understanding of the processes involved in the initiation and progression of atherosclerosis, ending in the rupture of the atherosclerotic plaque and the formation of a thrombus is intimately entwined in the mechanisms by which nutritional intake influences cardiovascular disease. The initiating event in atherosclerosis involves the induction of endothelial dysfunction by athero genic triggers, the best recognized of which are modified or oxidized LDL and the ad-vanced glycation end products that occur in diabetes ${ }^{27}$ This functional alteration in turn causes the increased expression of atherogenic signal molecules, including adhesion molecules, such as vascular cell adhesion molecule 1 (VCAM-1); chemoattractants such as monocyte chemoattractant protein 1 (MCP-1); and a host of growth factors and cytokines, among which are macrophage colony stimulating factor, CD40 ligand (CD154), interferon, tumor necrosis factor(TNF-), interleukin1 (IL-1), and IL-6. These signaling molecules allow the adhesion of monocytes and $\mathrm{T}$ lymphocytes to the arterial endothelium and their penetration into the intima. Monocytes undergo transformation and replication into macrophages and ultimately form the lipid-rich foam cells that are characteristic of the fatty streak, the first morphologically recognizable precursor of the atherosclerotic plaque. The same signal molecules are also responsible for the growth and the eventual destabilization of the plaque, being able to promote plaque rupture and the thrombogenic nature of the plaque content itself through the increase expression of molecules such as tissue factor. ${ }^{11}$

The above-listed signal molecules are mostly products of activated macrophages, lymphocytes, and endothelial cells, and they are inducible by cytokines. ${ }^{24}$ Therefore, they can be considered secondary mediators of local vascular inflammation. Resident macrophages perpetuate the cycle of local inflammation by releasing inflammatory cytokines, which result in the production of matrix-degrading metallo proteinases that contribute to plaque rupture, and tissue factor, which increases plaque thrombogenicity. ${ }^{11}$

\section{Rage polymorphisms}

RAGE diversity generated by alternative splicing is now welldocumented. ${ }^{28}$ These polymorphisms have been reported within exons, introns and gene regulatory regions. Some of them may affect not only the transcriptional activity, but also the binding affinity to RAGE ligands. Of particular importance, the single nucleotide polymorphisms, $-429 \mathrm{~T}>\mathrm{C}$ and $-374 \mathrm{~T}>\mathrm{A}$ of the promoter region of RAGE gene increase its expression two- and three-fold respectively, at least in vitro conditions. ${ }^{11}$

Most of the RAGE polymorphisms described have been associated with the onset of diseases or increased susceptibility risks. Many of these studies were carried out when the role of RAGE as a pattern recognition receptor was not clearly established, but in the light of the current knowledge, RAGE polymorphisms may represent new insights into the putative connection of genetically-determined hyper activities of the RAGE axis and the onset of immune diseases and prompt further investigations ${ }^{29}$ sRAGE may contribute to the removal/ detoxification of a diverse repertoire of pro-inflammatory ligands that are implicated in human diseases. The potential significance of circulating sRAGE is being investigated in a variety of pathological conditions through clinical research studies. Thus, the decoy function of sRAGE suggests the presence of a regulatory negative feedback mechanism in which sRAGE can serve to prevent the activation of cell surface RAGE. ${ }^{30}$ 


\section{Rage polymorphisms and cardiovascular disease}

Our laboratory group extensively studied one of the coding change polymorphisms of RAGE, the G82S variant. This variant, within the major site of ligand binding, the V-type immunoglobulin domain, when expressed in cells, alters ligand (S100) affinity and increased ligandstimulated generation of cytokines and matrix metalloproteinases. ${ }^{31}$ However, the variant is relatively uncommon and even when we analyzed the Framingham offspring cohort, we were unable to identify any associations of this variant with cardiovascular disease. ${ }^{32}$

Outside of the coding region, it is likely that variants in the promoter of RAGE may contribute to prediction for cardiovascular disease. For example, the 374T3 A variant has been shown to be protective against the development of cardiovascular disease (T/A or A/A individuals) in both diabetic and nondiabetic individuals. Another variant in the promoter region, $429 \mathrm{~T} 3 \mathrm{C}$, has also been studied. ${ }^{33}$

Unlike the 374T3A variant, however, no association has been reported with cardiovascular disease. 97 Importantly, however, many of the reported studies on RAGE polymorphisms in relation to cardiovascular disease are small. Furthermore, it is possible that haplotype analyses may be more informative in predicting the vulnerability to vascular disease, in the absence or presence of diabetes.Taken together, evidence is mounting from both experimental model systems and human studies (tissue expression levels, sRAGEs and polymorphisms) to suggest that the RAGE axis is highly relevant to human disease. ${ }^{34}$

Diabetes is also associated with a significant acceleration of atherosclerosis ${ }^{35,36}$ That this is at least partially due to glycation was already demonstrated by an early study by Brownlee ${ }^{37}$ Pharmacological inhibition of AGE formation using aminoguanidine resulted in reduced cross linking of proteins in arterial walls. Vice versa, the administration of exogenous AGEs to levels found in diabetics, induced atheroma formation in rabbits. ${ }^{38}$ These data are further corroborated by the expression pattern of receptors of AGEs in atherosclerotic plaques. CD36 is expressed on macrophages as a major receptor for oxidised $\mathrm{LDL}^{39} \mathrm{CD} 36$ is expressed in atherosclerotic lesions and considered to trigger the formation of macrophages into foam cells, a major event in the development of atherosclerosis. AGE-binding to CD36 can further accelerate this process by triggering tyrosine phosphorylation and NFkB activation. SRBI is essential for the reverse cholesterol transport as HDL. SRBI recognises AGEs and binding of AGE interferes with the uptake of acetylated HDL and suppresses SRBI-mediated efflux of cholesterol from cells..$^{40}$ Epidemiological studies demonstrated that low levels of sRAGE in plasma are correlated with a higher risk for cardiovascular mortality. ${ }^{41}$ In another population study, it was shown that the AA/GA genotypes of the RAGE $+557 \mathrm{G}>$ A polymorphism are associated with a significantly decreased risk of coronary heart disease ${ }^{42}$ (Table 1).

Table I Comparison of previous studies of the RAGE -374T/A genotypes and cardiovascular diseases are short brief in following table

\begin{tabular}{|c|c|c|c|c|}
\hline \multirow[t]{2}{*}{ Author } & \multirow[t]{2}{*}{ Year } & Race & \multirow[t]{2}{*}{ Polymorphism } & \multirow[t]{2}{*}{ Outcome } \\
\hline & & $\begin{array}{l}\text { Studies reporting } \\
\text { A positive } \\
\text { association }\end{array}$ & & \\
\hline Falcone et al. & 2008 & Italian & $-374 \mathrm{~T} / \mathrm{A}$ & $\begin{array}{l}\text { In non-diabetic patients having this polymorphism (A allele) has a } \\
\text { protective role for developing CAD. }\end{array}$ \\
\hline Falcone et al. & 2005 & Italian & $-374 \mathrm{~T} / \mathrm{A}$ & $\begin{array}{l}\text { AA genotype is protective in cardiac events, such as vascular } \\
\text { stenosis, and diabetes. }\end{array}$ \\
\hline Falcone et al. & 2004 & Italian & $-374 T / A$ & A allele has a protective effect in CAD. \\
\hline $\begin{array}{l}\text { Pettersson Fernholm } \\
\text { et al. }\end{array}$ & 2003 & Finnish & $-374 \mathrm{~T} / \mathrm{A}$ & $\begin{array}{l}\text { In CAD, acute } \mathrm{Ml} \text { and peripheral vascular disease patients, } \mathrm{A} \\
\text { allele is protective. }\end{array}$ \\
\hline Hudson et al. ${ }^{24}$ & 2001 & English & $-374 \mathrm{~T} / \mathrm{A}$ & $\begin{array}{l}\text { A allele down-regulates the transcription of RAGE } \\
\text { in diabetic retinopathy patients. }\end{array}$ \\
\hline Santos et al. & 2005 & $\begin{array}{l}\text { Africans//White } \\
\text { Brazilians }\end{array}$ & $-374 \mathrm{~T} / \mathrm{A}$ & $\begin{array}{l}\text { This polymorphism decreases the risk of ischemic heart disease } \\
\text { in African-Brazilians, but no association in White Brazilians. }\end{array}$ \\
\hline \multirow[t]{2}{*}{ Zee et al. ${ }^{30}$} & 2006 & Caucasian & & $\begin{array}{l}\text { The patients carrying }-374 T>A \text { variant have less ratio of } \\
\text { ischemic stroke }\end{array}$ \\
\hline & & $\begin{array}{l}\text { Studies reporting } \\
\text { negative } \\
\text { association }\end{array}$ & & \\
\hline Hudson et al. ${ }^{24}$ & 2001 & English & $-374 \mathrm{~T} / \mathrm{A}$ & $\begin{array}{l}\text { No association between diabetic and non-diabetic macrovascular } \\
\text { diseases and -374/A polymorphism }\end{array}$ \\
\hline Kirbis et al. & 2004 & Slovenian & $\begin{array}{l}-374 \mathrm{~T} / \mathrm{A} \text { and } \\
-429 \mathrm{~T} / \mathrm{C}\end{array}$ & No association with diabetic CAD \\
\hline Jixiong et al. & 2003 & Chinese & $\begin{array}{l}-374 T / A \text { and } \\
-429 T / C\end{array}$ & No association with diabetic retinopathy. \\
\hline
\end{tabular}

CAD, Coronary heart disease; CAD, Coronary artery disease; MI, Myocardial infarction; NIDDM, Non-insulin dependent diabetes mellitus 


\section{Conclusion}

In conclusion, the 63-bp ins/del, the $-374 \mathrm{~T}>\mathrm{A}$ and the $-429 \mathrm{~T}$ $>\mathrm{C}$ polymorphisms in the RAGE gene were not associated with susceptibility of HF and all-cause mortality in Caucasian-Brazilian HF patients. However, among African-Brazilians, the del allele (63bp ins/del polymorphism) was associated with a decreased risk of HF. To our knowledge, as this is the first study aimed to investigate the association of RAGE gene polymorphisms with HF, their role in the pathogenesis of HF cannot yet be elucidated. Taking into account the importance of RAGE for cardiac function and its potential use as a biomarker of prognosis in HF patients, further studies are needed to explore and clarify these associations. The term advanced glycation end products represents a variety of structurally highly diverse compounds. As reactive carbohydrates are the major cause of the AGE formation reaction, AGEs are on the one hand closely related to diabetes and on the other hand, are discussed to be a major cause of diabetes associated diseases. They can either be formed endogenously or incorporated from food, and are often associated with disease progression. Modulating AGE-formation and deposition is therefore a promising tool in treatment or prevention of several diseases ranging from diabetic complications over atherosclerosis to cancer and also ageing. In case of AGE-rich nutrition, the antioxidative potential of melanoidins might have positive effects, but further research on this topic is needed to identify components of promising pharmacological value.

\section{Acknowledgements}

None.

\section{Conflict of interest}

Authors declare there is no conflict of interest in publishing the article.

\section{References}

1. Zintzaras E, Zdoukopoulos N. A field synopsis and meta-analysis of genetic association studies in peripheral arterial disease: The CUMAGAS-PAD database. Am J Epidemiol. 2009;170(1):1-11.

2. Cohen CR, Diel VB, La Porta VL, et al. Association study of polymorphisms in the receptor for advanced glycation end-products (RAGE) gene with susceptibility and prognosis of heart failure. Gene. 2012;510(1):7-13.

3. Perez de Ciriza C, Lawrie A, Varo N. Osteoprotegerin in Cardiometabolic Disorders. Int J Endocrinol. 2015;2015(2015):1-15.

4. Rodino-Janeiro BK, Salgado-Somoza A, Teijeira-Fernandez E, et al. Receptor for advanced glycation end-products expression in subcutaneous adipose tissue is related to coronary artery disease. Eur $J$ Endocrinol. 2011;164(4):529-537.

5. Park JH, Li L, Choi JW, et al. The Association of $-429 \mathrm{~T}>\mathrm{C}$ and $-374 \mathrm{~T}>\mathrm{A}$ Polymorphisms in the RAGE Gene with Polycystic Ovary Syndrome. Int J Med Sci. 2016;13(6):451-456.

6. Cui X, Chen H, Hou X, et al. Polymorphism of the RAGE affects the serum inflammatory levels and risk of ischemic stroke in a Chinese population. Cell Physiol Biochem. 2013;32(4):986-996.

7. De Jonge P, Rosmalen JG, Kema IP, et al. Psychophysiological biomarkers explaining the association between depression and prognosis in coronary artery patients: a critical review of the literature. Neurosci Biobehav Rev. 2010;35(1):84-90.
8. Davis, Davis RC, Hobbs FDR, et al. ABC of heart failure: history and epidemiology. BMJ. 2000;320(7226):39-42.

9. Kalea, Anastasia Z, Ann M Schmidt, et al. RAGE: a novel biological and genetic marker for vascular disease. Clin Sci (Lond). 2009;116(8):621-637.

10. Yan SF, Ramasamy R, Schmidt AM. The receptor for advanced glycation endproducts and cardiovascular disease. Expert Rev Mol Med. 2009; 11:e9.

11. Kalea AZ, Schmidt AM, Hudson BI. RAGE: a novel biological and genetic marker for vascular disease. Clin Sci. 2009;116(8):621-637.

12. Koyama Y, Takeishi Y, Suzuki S. Soluble receptor for advanced glycation endproducts is a prognostic factor for heart failure. $J$ Card Fail. 2008;14:133-139.

13. Raposeiras-Roubín S, Rodiño-Janeiro BK, Grigorian-Shamagian L, et al Relation of Soluble Receptor for Advanced Glycation End Products to Predict Mortality in Patients With Chronic Heart Failure Independently of Seattle Heart Failure Score. Am J Cardiol. 2011;107:938-44.

14. Raposeiras-Roubín, Raposeiras-Roubín S. Soluble receptor of advanced glycation end products levels are related to ischaemic aetiology and extent of coronary disease in chronic heart failure patients, independent of advanced glycation end products levels: new roles for soluble RAGE Eur. J Heart Fail. 2010;12(2010):1092-1100.

15. Wang, Wang LJ, Lu L, et al. Increased serum high-mobility group box-1 and cleaved receptor for advanced glycation endproducts levels and decreased endogenous secretory receptor for advanced glycation endproducts levels in diabetic and non-diabetic patients with heart failure Eur. J Heart Fail. 2011;13(4):440-449.

16. Hofmann MA, Drury $\mathrm{S}, \mathrm{Fu} \mathrm{C}$, et al. RAGE mediates a novel proinflammatory axis: a central cell surface receptor for S100/calgranulin polypeptides. Cell. 1999;97(7):889-901.

17. Wong FN, Chua KH, Kuppusamy UR, et al. Association of the receptor for advanced glycation end-products (RAGE) gene polymorphisms in Malaysian patients with chronic kidney disease. PeerJ. 2016;4:e1908.

18. Upadhyay RK. Emerging risk biomarkers in cardiovascular diseases and disorders. J Lipids. 2015;971453.

19. Mokbel A, Rashid L, Al-Harizy R. Decreased level of soluble receptors of advanced glycated end products (sRAGE) and glycine82serine (G82S) polymorphism in Egyptian patients with RA. The Egyptian Rheumatologist. 2011;33(1):53-60.

20. Gonzalez I, Romero J, Rodriguez BL, et al. The immunobiology of the receptor of advanced glycation end-products: trends and challenges. Immunobiology. 2013;218(5):790-797.

21. Pan H, He L, Wang B, et al. The relationship between RAGE gene four common polymorphisms and breast cancer risk in northeastern Han Chinese. Sci Rep. 2014;4:4355.

22. Hofmann MA, Yang Q, Harja E, et al. The RAGE Gly82Ser polymorphism is not associated with cardiovascular disease in the Framingham offspring study. Atherosclerosis. 2005;182(2):301-305.

23. Giuseppina Basta, Ann Marie Schmidt, Raffaele De Caterina. Advanced glycation end products and vascular inflammation: implications for accelerated atherosclerosis in diabetes. Cardiovascular Res. 2004;63(4):582-592.

24. Hudson BI, Harja E, Moser B, et al. Soluble levels of receptor for advanced glycation endproducts (sRAGE) and coronary artery disease: the next C-reactive protein?. Arterioscler Thromb Vasc Biol. 2005;25(5):879-882. 
25. Rojas A, Delgado-Lope F, Gonzalez, et al. The receptor for advanced glycation end-products: a complex signaling scenario for a promiscuous receptor. Cell Signal. 2013;25(3):609-614.

26. Sorci G, Riuzzi F, Giambanco I, et al. RAGE in tissue homeostasis, repair and regeneration. Biochim Biophys Acta. 2013;1833(1):101-109.

27. Yan SF, Ramasamy R, Schmidt AM. The RAGE axis: a fundamental mechanism signaling danger to the vulnerable vasculature. Circ Res. 2010;106(5):842-853.

28. Kalousova M, Zima T, Tesar V, et al. Advanced glycoxidation end products in chronic diseases-clinical chemistry and genetic background. Mutat Res. 2005;579(1-2):37-46.

29. Kouidrat Y, Amad A, Arai M, et al. Advanced glycation end products and schizophrenia: A systematic review. J Psychiatr Res. 2015;66(67):112-117.

30. Zee RYL, Romero JR, Gould JL, et al. PM: Polymorphisms in the advanced glycosylation end product- specific receptor gene and risk of incident myocardial infarction or ischemic stroke. Stroke. 2006;37(7):1686-1690.

31. Laine MLBG, Loos BG, W Crielaard. Gene polymorphisms in chronic periodontitis. Int J Dent. 2010;324719:1-22.

32. Liu W, Ge S, Liu Y, et al. Polymorphisms in three genes are associated with hemorrhagic stroke. Brain Behav. 2015;5(11):e00395.

33. Mahajan N, V Dhawan. Receptor for advanced glycation end products (RAGE) in vascular and inflammatory diseases. Int $J$ Cardiol. 2013;168(3):1788-1794.
34. Abe R, Yamagishi S. AGE-RAGE system and carcinogenesis. Curr Pharm Des. 2008;14(10):940-945.

35. T Mazzone. Adipose tissue and the vessel wall Curr Drug Targets. 2007;8(6):1190-1195.

36. Mazzone T, Chait A, Plutzky J. Cardiovascular disease risk in type 2 diabetes mellitus: insights from mechanistic studies. Lancet. 2008;371(3626):1800-1809.

37. Brownlee M, Vlassara H, Kooney A, et al. Cerami Aminoguanidine prevents diabetes-induced arterial wall protein cross-linking. Science. 1986;232(4758):1629-1632.

38. Vlassara H, Fuh H, Donnelly T, et al. Cybulsky Advanced glycation endproducts promote adhesion molecule (VCAM-1, ICAM-1) expression and atheroma formation in normal rabbits. Mol Med. 1995;1(4):447-456.

39. Endemann G, StantonLW, Madden KS, etal.CD36 is a receptor for oxidized low density lipoprotein. J Biol Chem. 1993;268(16):11811-11816.

40. Ohgami N, Miyazaki A, Sakai M, et al. Advanced Glycation End Products(AGE) Inhibit Scavenger Receptor Class B type I-Mediated Reverse Cholesterol Transport: A New Crossroad of AGE to Cholesterol Metabolism. Journal of atherosclerosis and thrombosis. 2003;10:1-6.

41. H Koyama, T Shoji, S Fukumoto, et al. Low circulating endogenous secretory receptor for AGEs predicts cardiovascular mortality in patients with end-stage renal disease. Arterioscler Thromb Vasc Biol. 2007;27:147-153.

42. Yoon SJ, Park S, Shim CY, et al. Association of RAGE gene polymorphisms with coronary artery disease in the Korean population. Coron Artery Dis. 2007;18(1):1-8. 